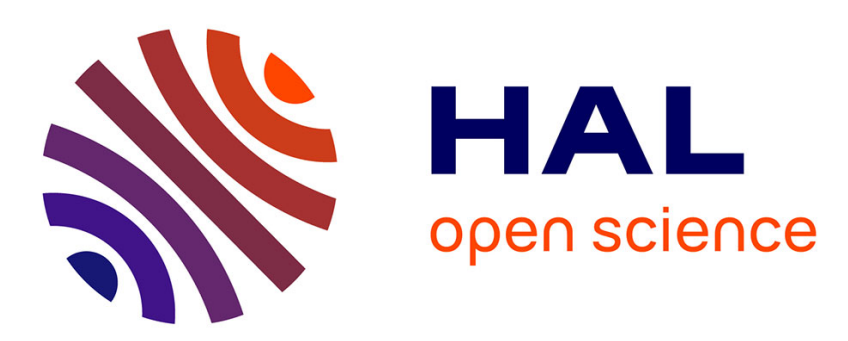

\title{
Line-shape and resolution enhancement of high-resolution F.T.N.M.R. in an inhomogeneous magnetic field
}

\author{
J. Taquin
}

\section{- To cite this version:}

J. Taquin. Line-shape and resolution enhancement of high-resolution F.T.N.M.R. in an inhomogeneous magnetic field. Revue de Physique Appliquée, 1979, 14 (6), pp.669-681. 10.1051/rphysap:01979001406066900 . jpa-00244646

\section{HAL Id: jpa-00244646 https://hal.science/jpa-00244646}

Submitted on 1 Jan 1979

HAL is a multi-disciplinary open access archive for the deposit and dissemination of scientific research documents, whether they are published or not. The documents may come from teaching and research institutions in France or abroad, or from public or private research centers.
L'archive ouverte pluridisciplinaire HAL, est destinée au dépôt et à la diffusion de documents scientifiques de niveau recherche, publiés ou non, émanant des établissements d'enseignement et de recherche français ou étrangers, des laboratoires publics ou privés. 


\title{
Line-shape and resolution enhancement of high-resolution F.T.N.M.R. in an inhomogeneous magnetic field
}

\author{
J. Taquin \\ Institut d'Electronique Fondamentale (*), Université Paris XI, Bât. 220, 91405 Orsay, France
}

(Reçu le 25 janvier 1979, accepté le 27 février 1979)

\begin{abstract}
Résumé. - L'influence de l'inhomogénie spatiale du champ magnétique est étudiée, à l'aide des équations de Bloch, par la déformation produite sur une raie théoriquement lorentzienne. L'étude est effectuée pour un échantillon immobile dans différents cas de détection et sur le signal de précession libre dans le cas d'un échantillon tournant. La mise en évidence d'une fonction ne dépendant que de la forme de l'échantillon et que de l'inhomogénie du champ magnétique principal permet de proposer une méthode d'amélioration de la résolution des spectres de résonance magnétique nucléaire par transformée de Fourier. Cette méthode, valable pour des composés liquides dont les raies sont théoriquement lorentziennes, n'introduit pas de facteur empirique. Quelques exemples permettent d'en apprécier l'efficacité.
\end{abstract}

\begin{abstract}
The spatial inhomogeneous magnetic field is studied by means of its effects on a line shape which theoretically would be a lorentzian curve. The Bloch equations permit to solve this problem for a non-spinning sample, in various experiments, and also for a sample rotating around the axis of the field, in the case of a free induction decay signal. We show as an example, how one can theoretically obtain the line shape for some types of inhomogeneous fields.

Then, through the definition of a complex function depending only on the inhomogeneity of the magnetic field and on the form of the sample, we propose a new method for the resolution enhancement of Fourier transform nuclear magnetic resonance. This method, suitable for lines with a theoretically lorentzian shape, does not use an empirical parameter. Few examples are presented to demonstrate the potential of this method.
\end{abstract}

1. Introduction. - In N.M.R. we know that the line shape is a function of the inhomogeneity of the magnetic field. We are going to show that a detailed study of the effects gives a simple expression which only depends on the inhomogeneity of the magnetic field and on the shape of the sample.

This relation can have many applications. We will demonstrate one of them in this paper : the resolution enhancement of a spectrum; other possible applications are : a systematic study of the magnetic field configuration, or the precise measure of the frequency of a resonance line in relation to the standard frequency of the spectrometer, because frequency shifts introduced by the inhomogeneity of the field can be compensated.

First we evaluate the contribution of the inhomogeneity of the magnetic field to the line shape. We obtain, whatever method of N.M.R. detection is used, the same mathematical expression provided that some experimental conditions are satisfied [1].

$\left(^{*}\right)$ Laboratoire associé au C.N.R.S.
We then give some examples of resolution enhancement.

In high resolution N.M.R. spectroscopy, the line shape is lorentzian often because it is defined by two constants (longitudinal time $T_{1}$ and transversal time $\left.T_{2}\right)$. The observed times $T_{1}$ and $T_{2}$ are in general smaller than the natural times used in the equations and we can use the phenomenological Bloch's equations to describe the experiment.

As we can translate, with appropriate conditions, into a mathematical F.T.N.M.R. form many of the methods of NMR detection we are going to use the free induction decay signal for a $90^{\circ}$ pulse for the examples.

We then obtain the transverse magnetization for one line :

$$
M(t)=j M(0) \mathrm{e}^{-t / T_{2}} \mathrm{e}^{-j \Delta \omega t}
$$

in which $t=0$ is the time at the end of the pulse, $\Delta \omega=\omega_{0}-\omega$, in which $\omega_{0}=\gamma H_{0}$, is the Larmor frequency. 
Under appropriate conditions $[2,3,4]$, we can write :

$$
M_{x}(0)=0 \text { and } M_{y}(0)=M_{0} \sin \gamma H_{1} \tau \approx M_{0}
$$

for a $90^{\circ}$ pulse, i.e. :

$$
\tau=\frac{\pi}{2 \gamma H_{1}}
$$

Finally we have :

and

$$
M_{y}(t)=M_{0} \mathrm{e}^{-t / T_{2}} \cos \Delta \omega t
$$

$$
M_{x}(t)=-M_{0} \mathrm{e}^{-t / T_{2}} \sin \Delta \omega t .
$$

The Fourier transform of this expression gives the complex spectrum :

$$
M(\omega)=M_{0} \frac{T_{2}}{1+j \Delta \omega T_{2}}
$$

whose real part is lorentzian.

The factor $M(0)$, only, is modified when we use most another method of detection.

We are going to investigate successively :

- the influence of a static inhomogeneous field ;

- the influence of a spinning sample ;

- some examples of signals in a defined inhomogeneous field;

- a new method of resolution enhancement, using the inhomogeneity functions defined below, with some experiments.

2. Static inhomogeneous field. - We assume here, to a first approximation and to make the presentation simpler, that the transverse radio frequency field $H_{1}$ is homogeneous over the whole volume of the resonant coil. If it is not, $M(0)$ will depend on the three spatial coordinates. We will described late on the modifications which are necessary for inhomogeneous.

2.1 NeW ASPECT OF THE FORMULAS. - If the static magnetic field is not homogeneous over the volume of the sample, it is possible to write :

$$
\mathbf{H}=\left[H_{0}+h_{z}(v)\right] \mathbf{k}+h_{x}(v) \mathbf{i}+h_{y}(v) \mathbf{j}
$$

in which $v$ holds for the three spatial coordinates $(x, y, z) . H_{0}$ is much larger than $h_{x}, h_{y}$ and $h_{z}$ and it is possible to neglect $h_{x}$ and $h_{y}$.

This leads to the resonance offset

$$
\Delta \omega=\gamma H_{0}-\omega+\gamma h_{z}(v) .
$$

Equations (1) and (2), which show the line shape, cannot be used now for the whole volume of the irradiated sample. But, if the spin density is uniform, for a small part of the volume $\mathrm{d} v$ around a point with coordinates, $v_{0}\left(x_{0}, y_{0}, z_{0}\right)$ which the volume being considered to contain a very large number of spins, then nothing is modified in the preceding solution if we replace $\gamma H_{0}-\omega$ by

$$
\gamma H_{0}-\omega+\gamma h_{z}\left(v_{0}\right)=\Delta \omega+\gamma h_{z}\left(v_{0}\right) .
$$

For all the spins of the system it is then sufficient to integrate these expressions over the whole volume of the irradiated sample, i.e. the part of the sample included in the cavity.

Then we have for the free induction decay signal $[1,5]$ :

$$
\bar{M}(t)=j M_{0} \mathrm{e}^{-t / T_{2}} \int_{V} \mathrm{e}^{-\tau\left[\Delta \omega+\gamma h_{z}(v)\right] t} \mathrm{~d} v
$$

in this expression $\int_{V}$ represents a triple integral over the whole volume of the irradiated sample. The line shape is no longer lorentzian and depends on the shape of the sample.

For instance, with a cylindrical sample $2 d$ in height, $R$ in radius, symmetrically placed over the origin of the coordinates, we have for the free induction decay :

$$
\begin{aligned}
\bar{M}(t)= & j M_{0} \mathrm{e}^{-t / T_{2}} \int_{-d}^{d} \int_{0}^{R} \int_{0}^{2 \pi} \times \\
& \times \mathrm{e}^{-j\left[\Delta \omega+\gamma h_{z}(z, \rho \cos \theta, \rho \sin \theta)\right] t} \rho \mathrm{d} \rho \mathrm{d} z \mathrm{~d} \theta .
\end{aligned}
$$

When the transverse field $H_{1}$ cannot be considered as homogeneous over the whole irradiated volume, we must change $M_{0}$ into $M g(v)$ where $g(v)$ is a positive function of the three space coordinates. $g(v)$ depends on the development of $H_{1}$ and it is zero outside the irradiated volume of the sample. Equation (3) must then be replaced by :

$$
\bar{M}(t)=j M \mathrm{e}^{-t / T_{2}} \int_{V} g(v) \mathrm{e}^{-j\left[\Delta \omega+\gamma h_{z}(v)\right] t} \mathrm{~d} v .
$$

2.2 THE FIELD EXPRESSION. - The term $h_{z}$ is the development of the $z$ component around a central point, which is origin of the coordinates where $h_{z}=0$. Such a development has been used to calculate and build correcting coils, in order to correct the various gradients of the field. According to the relation $\operatorname{div} \mu \mathbf{H}=0$, the magnetic field can be developed by using independent gradients $\left(a_{\mathrm{i}}\right)$, the first twelve terms being [6] :

$$
\begin{aligned}
h_{z}(x, y, z)= & a_{1} z+a_{2} x+a_{3} y+a_{4}\left(2 z^{2}-x^{2}-y^{2}\right)+a_{5} z x+a_{6} z y+ \\
& +a_{7}\left(x^{2}-y^{2}\right)+a_{8} x y+a_{9}\left[z^{3}-\frac{3}{2} z\left(x^{2}+y^{2}\right)\right]+a_{10} x\left(4 z^{2}-x^{2}-y^{2}\right) \\
& +a_{11} y\left(4 z^{2}-x^{2}-y^{2}\right)+a_{12}\left[z^{4}-3 z^{2}\left(x^{2}+y^{2}\right)+\frac{3}{8}\left(x^{2}+y^{2}\right)^{2}\right]+\cdots .
\end{aligned}
$$


Even in the case of cylindrical geometry (spectrometers built with a supraconducting coil), the cartesian coordinates are generally used to locate these coils in the laboratory axis. The twelve gradients can be corrected by twelve independent coils.

This development, introduced in the preceding expressions, allows us to find the deformation of the line shape caused by each of the gradients depending on the value of $a_{\mathrm{i}}$. This operator is not linear and the deformation of the line caused by any of the gradients depends on the other gradient values.

This remark explains why experimentally it is difficult to obtain an homogeneous magnetic field with such correcting coils; all the coils must be used to try and obtain the best result.

Automatic corrections of the inhomogeneity of the magnetic field in the case of one or two gradients have been used [7]; for long duration experiments, such systems can be useful because of the slow variation of the value of the parameters $a_{\mathrm{i}}$. However, the influence of high order gradients cannot be neglected, as we will see later on, and these automatic methods are not commonly used.

3. Spinning sample. - In order to obtain an important resolution enhancement, the sample is usually made to spin around the $z$-axis of a superconducting coil.

The field $H_{z}=H_{0}+h_{z}$ can still be written :

$$
H_{z}=\sum_{m n p}^{\infty} A_{m n p} x^{m} y^{n} z^{p}
$$

in which the parameters $A_{m n p}$ depend on one or more independent gradients.

With cylindrical coordinates $x=\rho \cos \theta$ and $y=\rho \sin \theta$ we have :

$$
H_{z}=\sum_{m n p}^{\infty} A_{m n p} z^{p} \rho^{m+n} \cos ^{m} \theta \cdot \sin ^{n} \theta
$$

$\cos ^{m} \theta, \sin ^{n} \theta$ and their products can be split into a series of terms in $\cos N \theta$ and $\sin N \theta$ with

$$
0 \leqslant N \leqslant m+n
$$

and after rearrangement

$$
\begin{aligned}
& H_{z}=\sum_{N=0}^{\infty} A_{N}(\rho, z) \cos N \theta+B_{N}(\rho, z) \sin N \theta= \\
&=\sum_{N=0}^{\infty} H_{N}(\rho, z) \cos \left[N \theta-\varphi_{N}(\rho, z)\right]
\end{aligned}
$$

or :

$$
H_{z}=H_{0}+\sum_{N=0}^{\infty} h_{N}(\rho, z) \cos \left[N \theta-\varphi_{N}(\rho, z)\right]
$$

if we isolate the constant term. In these expressions we have :

$h_{N}(\rho, z)=\sqrt{A_{N}^{2}+B_{N}^{2}}$ and $\varphi_{N}(\rho, z)=\operatorname{arctg} \frac{B_{N}}{A_{N}}$.

For gradients with orders $N \leqslant 2$, i.e. the first 8 parameters $a_{\mathrm{i}}$ of the equation (6) development we can obtain the following relations :

$$
\begin{aligned}
& h_{0}(\rho, z)=a_{1} z+2 a_{4} z^{2}-a_{4} \rho^{2} \\
& \varphi_{0}(\rho, z)=0 \\
& h_{1}(\rho, z)=\rho \sqrt{\left(a_{2}+a_{5} z\right)^{2}+\left(a_{3}+a_{6} z\right)^{2}} \\
& \varphi_{1}(\rho, z)=\operatorname{arctg} \frac{a_{3}+a_{6} z}{a_{2}+a_{5} z} \\
& h_{2}(\rho, z)=\rho^{2} \sqrt{a_{7}^{2}+a_{8}^{2} / 4} \\
& \varphi_{2}(\rho, z)=\operatorname{arctg} a_{8} / 2 a_{7} .
\end{aligned}
$$

With the sample spinning around the $z$-axis at an angular speed $\Omega$ the spins in the volume $\mathrm{d} v$ with coordinates $(z, \rho, \theta)$ are moving along a circle with coordinates $(z, \rho, \theta+\Omega t)$. We can express the applied magnetic field as :

$H_{z}=H_{0}+\sum_{n=0}^{\infty} h_{n}(\rho, z) \cos \left[n \Omega t+n \theta-\varphi_{n}(\rho, z)\right]$.

The applied magnetic field can no longer be considered as a static magnetic field because of its inhomogeneity. The resolution of the Bloch's equations must be revised. We are going to do this for the case of free induction decay signals.

The pulse along the $x$-axis with a $2 H_{1 x}$ amplitude exits only during the time $\tau$ after which $H_{1 x}$ is null. Since $\tau$ is very small when compared to the period of rotation and the sample has practically not moved during this time, $H_{1 x}$ can be considered as a static term.

At the end of the pulse, the origin of time, $H_{1 x}$ is null and during the free induction decay, the Bloch's equations can be written in the following simplified form for this small volume $\mathrm{d} v$ :

$$
\begin{aligned}
& \frac{\partial M}{\partial t}+j M\left(\omega_{0}-\omega\right)+\frac{M}{T_{2}}=0 ; \\
& \frac{\partial M_{z}}{\partial t}+\frac{M_{z}}{T_{1}}=\frac{M_{0}}{T_{1}} .
\end{aligned}
$$

We can always write $M_{0}=\chi_{0} H_{z}=\chi_{0} H_{0}$ constant because $H_{0} \gg h(v, t)$. But we have :

$$
\begin{aligned}
\omega_{0}-\omega & =\gamma H_{0}-\omega+\gamma \sum_{n=0}^{\infty} h_{n}(\rho, z) \cos \left[n \Omega t+n \theta-\varphi_{n}(\rho, z)\right] \\
& =\gamma H_{0}-\omega+\gamma h_{0}(\rho, z)+\gamma \sum_{n=1}^{N} h_{n}(\rho, z) \cos \left[n \Omega t+n \theta-\varphi_{n}(\rho, z)\right]
\end{aligned}
$$

if we limit the development to $n_{\max }=N$. 
We write $\Delta \omega=\gamma H_{0}-\omega$ and also to simplify the expressions

$\Delta^{\prime} \omega=\Delta \omega+\gamma h_{0}(\rho, z), \quad h_{n}=h_{n}(\rho, z), \quad \zeta_{n}=\varphi_{n}(\rho, z)$.
These equations are of the same type as those encountered in the double field modulation [8] experiment and we can use the same type of solution. We obtain :

$$
\begin{aligned}
& M(t)=M_{x}(t)+j M_{y}(t)=K \exp \left\{-\int_{-\infty}^{t}\left[\frac{1}{T_{2}}+j \Delta^{\prime} \omega+j \gamma \sum_{n=1}^{N} h_{n} \cos (n \Omega t+n \theta-\zeta n)\right] \mathrm{d} t\right\} \\
& M(t)=K \mathrm{e}^{-t / T_{2}} \mathrm{e}^{-j \Delta^{\prime} \omega t} \exp \left[j \sum_{n=1}^{N} B_{n} \sin (n \Omega t+n \theta-\zeta n)\right]
\end{aligned}
$$

with

$$
B_{n}=-\frac{\gamma h_{n}}{n \Omega}
$$

in which $K$ is given by :

$$
M(0)=M_{x}(0)+j M_{y}(0)=j M_{0} \sin \delta \approx j M_{0} \sin \left(\gamma \tau H_{1 x}\right)=j M_{0} \mathbf{H}_{1 x} .
$$

At this stage, the possible inhomogeneity of $H_{1 x}$ should be taken into account. But, if we neglect it in the first approximation, we have :

$$
M(t)=j M_{0} \mathrm{e}^{-t / T_{2}} \mathbf{H}_{1 x} \mathrm{e}^{-j \Delta^{\prime} \omega t} \prod_{n^{\prime}=1}^{N} \mathrm{e}^{-j B_{n^{\prime}} \sin \left(n^{\prime} \theta-\zeta_{n^{\prime}}\right)} \prod_{n=1}^{N} \mathrm{e}^{j B_{n} \sin \left(n \Omega t+n \theta-\zeta_{n}\right)}
$$

where $\mathrm{H}_{1 x}=1$ for a $90^{\circ}$ pulse.

Then, by using the Bessel's functions with

$$
\mathrm{e}^{j x \sin y}=\sum_{n=-\infty}^{\infty} J_{n}(x) \cdot \mathrm{e}^{j n y}
$$

we obtain :

$$
M(t)=j M_{0} \mathrm{e}^{-t / T_{2}} \mathbf{H}_{1 x} \mathrm{e}^{-j \Delta^{\prime} \omega t} \prod_{n^{\prime}=1}^{N} \sum_{m^{\prime}} J_{m^{\prime}}\left(B_{n^{\prime}}\right) \mathrm{e}^{-j m^{\prime}\left(n^{\prime} \theta-\zeta_{n^{\prime}}\right)} \prod_{n=1}^{N} \sum_{m} J_{m}\left(B_{n}\right) \mathrm{e}^{j m\left(n \Omega t+n \theta-\zeta_{n}\right)}
$$

or :

$$
M(t)=j M_{0} \mathrm{e}^{-t / T_{2}} \mathbf{H}_{1 x} \mathrm{e}^{-j \Delta^{\prime} \omega t} \sum_{\substack{m_{1}, \ldots, m_{N} \\ m_{1}^{\prime}, \ldots, m_{N}^{\prime}}} \prod_{n=1}^{N}\left[J_{m_{n}}\left(B_{n}\right) J_{m_{n}^{\prime}}\left(B_{n}\right)\right] \exp \left[j \sum_{n=1}^{N} m_{n} n \Omega t+n\left(m_{n}-m_{n}^{\prime}\right) \theta-\zeta_{n}\left(m_{n}-m_{n}^{\prime}\right)\right] .
$$

With a good phase adjustment, we observe $M_{y}(t)$, i.e. the imaginary part of $M(t)$.

For all the irradiated samples we have :

$$
\bar{M}(t)=\int_{d_{1}}^{d_{2}} \mathrm{~d} z \int_{0}^{R} \rho \mathrm{d} \rho \int_{\theta_{1}(\rho)}^{\theta_{2}(\rho)} M(t) \mathrm{d} \theta
$$

in the general case, when the sample is not spinning in a symmetrical way. For a cylindrical sample placed at the origin of the development of the field, we have the simplified expression because of the influence of $\theta$. We then have :

$$
\bar{M}(t)=\int_{-d}^{d} \mathrm{~d} z \int_{0}^{R} \rho \mathrm{d} \rho \int_{0}^{2 \pi} M(t) \mathrm{d} \theta
$$

in which $\theta$ occurs in the form :

$$
\exp \left(j \sum_{n} \alpha_{n} \theta\right) .
$$

Because of the term in $\Omega t$ a spectrum with sidebands appears in experiments in which the magnetic field or frequency are modulated. However the result is different because the inhomogeneity of the magnetic field $H_{1 x}$ cannot be neglected for the side bands [1,9].

For the principal term we can show that the signal becomes :

$$
\begin{aligned}
\bar{M}(t)=2 \pi j M_{0} \mathbf{H}_{1 x} & \mathrm{e}^{-t / T_{2}} \int_{-d}^{d} \int_{0}^{R} \times \\
& \times \mathrm{e}^{-j\left[\Delta \omega+\gamma h_{0}(\rho, z)\right] t} \rho \mathrm{d} \rho \mathrm{d} z .
\end{aligned}
$$


This expression is similar to equation (3) but the triple integral is replaced by a double integral and the $h_{z}$ term is replaced by $h_{0}$.

For a second order development, the spinning of the sample allows us to neglect 6 of the 8 gradients which appear in equation (10). We also eliminate the coupling relations between gradients because of the nonlinear operator and finally only 2 gradients with $z$ and $z^{2}$ terms remain. This explains the spectacular resolution enhancement brought by the spinning sample method.

However the $x$ and $y$ directions still exist together with the $a_{4}$ parameter through the expression

$$
-a_{4} \sqrt{x^{2}+y^{2}}
$$

used to write the second order gradient (curvature).

If $H_{1 x}$ is not an homogeneous field, then we can show that the expression of the magnetic moment becomes with

$$
\begin{gathered}
\hat{H}_{1 x}=H_{10}+\sum_{p=-\infty}^{\infty} \frac{h_{1|p|}(\rho, z)}{2} \mathrm{e}^{-j \cdot \operatorname{sign}(p) \cdot \zeta_{1|p|}(\rho, z)} \\
\bar{M}(t)=K \mathrm{e}^{-t / T_{2}} \int_{-d_{1}}^{d_{2}} \mathrm{~d} z \int_{0}^{R} \hat{H}_{1 x} \prod_{n=1}^{\infty} J_{0}^{2}\left(\frac{\gamma h_{n}(\rho, z)}{n \Omega}\right) \times \\
\times \mathrm{e}^{-j\left[\Delta \omega+\gamma h_{0}(\rho, z)\right] t} \rho \mathrm{d} \rho .
\end{gathered}
$$

4. Signal in an inhomogeneous field. - Here, we will plot various aspects of the signal for various values of the different gradients.

The following curves show the theoretical line shape obtained using a computer, being the defect

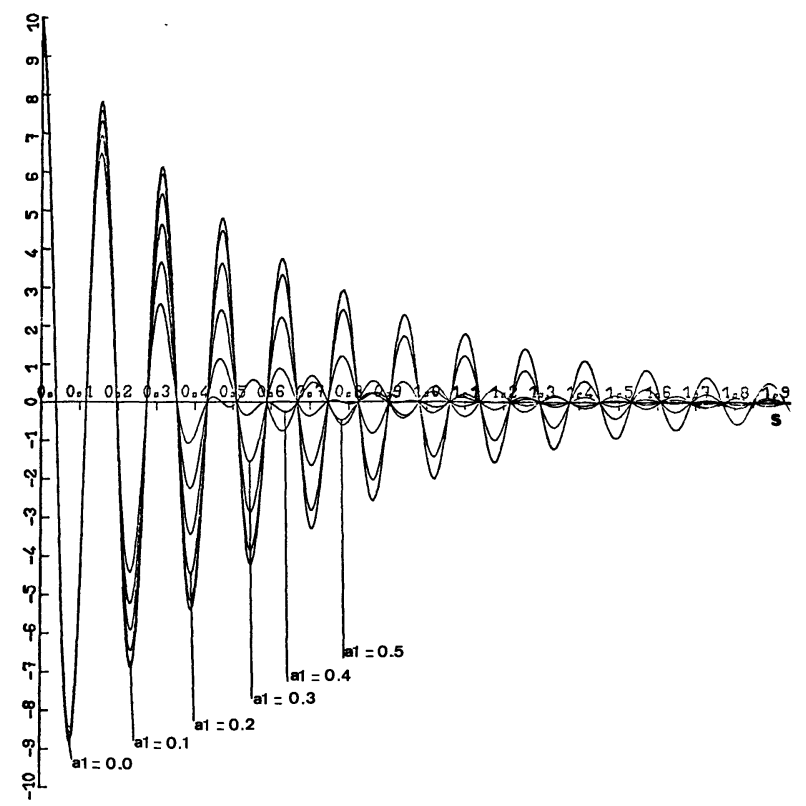

Fig. 1. - Free induction decay signal for the magnetic field

$$
H_{z}=H_{0}+a_{1} z \text {. }
$$

The curves $M_{y}(t)$ are drawn for different values of $a_{1}$ in $\mathrm{mG} / \mathrm{cm}$. We can see a beat pattern for large values of $a_{1}$.

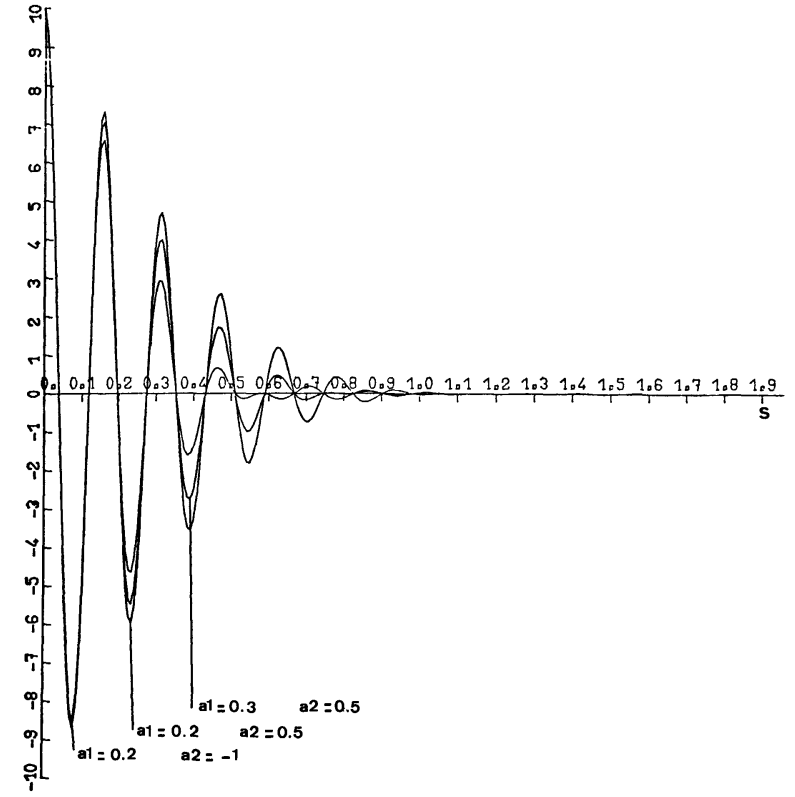

Fig. 2. - Free induction decay signal for a magnetic field of the form : $H_{z}=H_{0}+a_{1} z+a_{2} x$ for some values of the parameters $a_{1}$ and $a_{2}$ in $\mathrm{mG} / \mathrm{cm}$. The sample is supposed to be still; if it is spinning around the $z$-axis we obtain the signal shown in figure 1 .

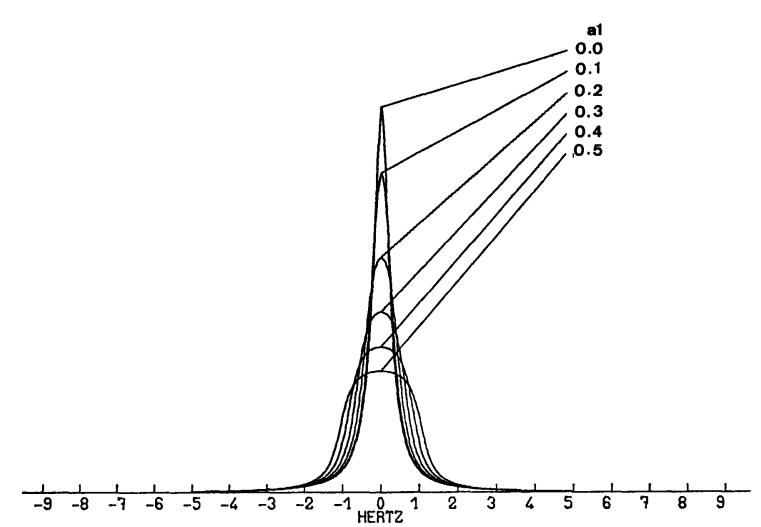

Fig. 3. - Fourier transform of the curve in figure 1.

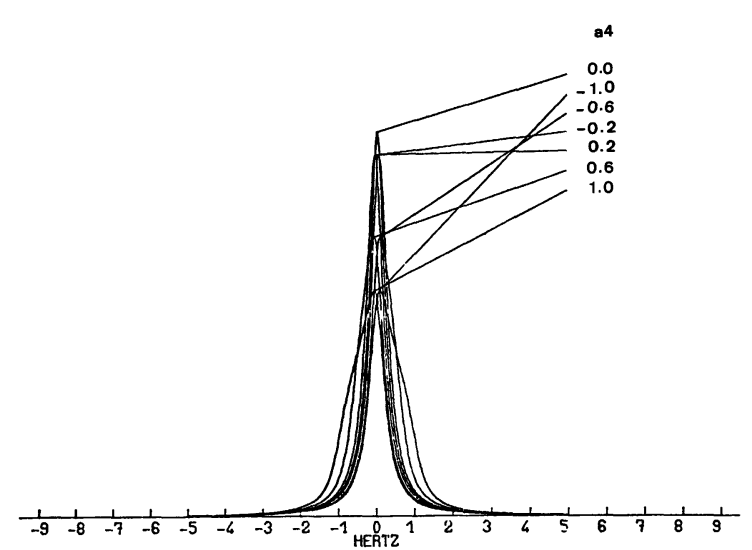

Fig. 4. - Spectrum for a magnetic field of the form :

$$
H_{z}=H_{0}+a_{4}\left[z^{2}-\frac{1}{2}\left(x^{2}+y^{2}\right)\right], a_{4} \text { in } \mathrm{mG} / \mathrm{cm}^{2} \text {. }
$$


simulated by introducing one specified gradient. The symmetrical sample is a cylinder $0.5 \mathrm{~cm}$ in radius. The field $H_{1}$ is perfectly homogeneous in the whole volume of the cavity $1 \mathrm{~cm}$ in height and zero elsewhere.

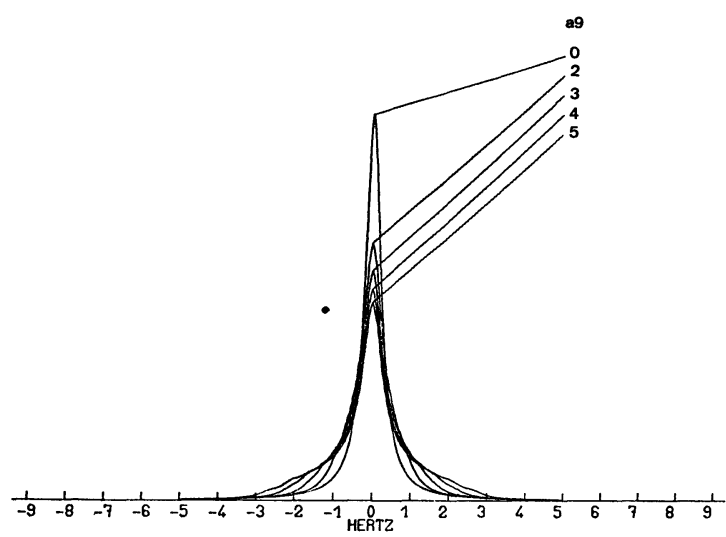

Fig. 5. - The magnetic field has the form :

$H_{z}=H_{0}+a_{9}\left[z^{3}-\frac{3}{2} z\left(x^{2}+y^{2}\right)\right], a_{9}$ in $\mathrm{mG} / \mathrm{cm}^{3}$.

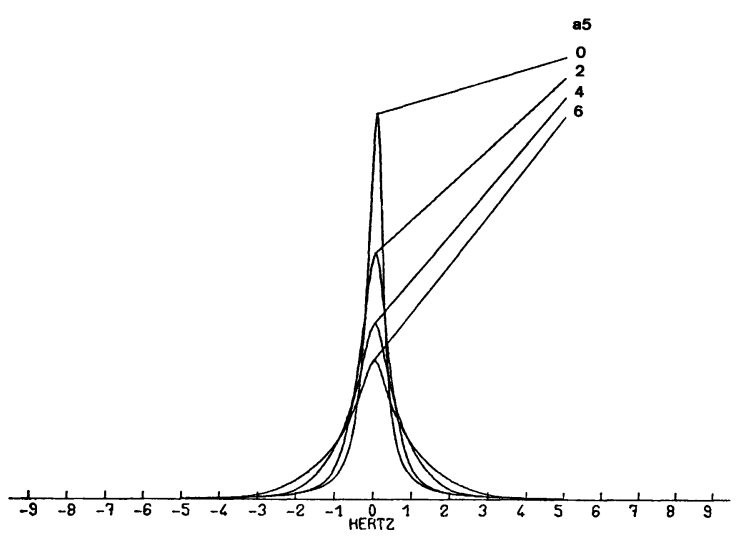

Fig. 6. - Spectrum for a magnetic field of the form :

$$
H_{z}=H_{0}+a_{5} x z, a_{5} \text { in } \mathrm{mG} / \mathrm{cm}^{2} .
$$

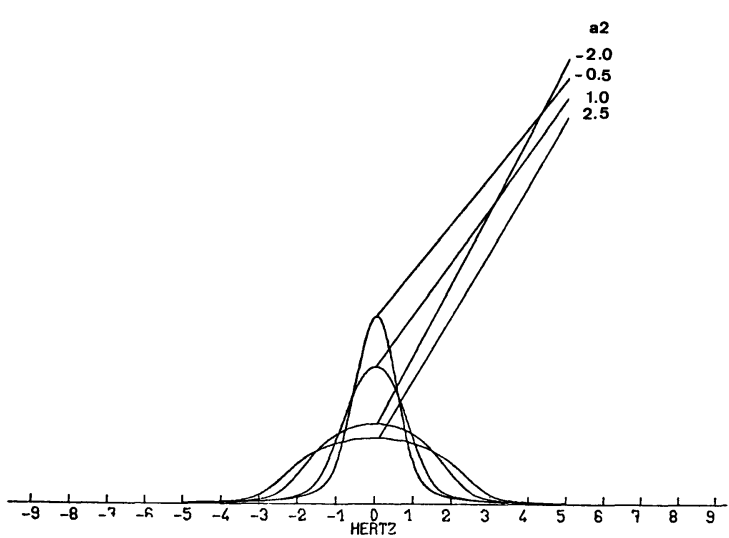

Fig. 7. - Spectrum for a magnetic field with two parameters $a_{1}$ and $a_{2}$ :

$$
H_{z}=H_{0}+0.25 z+a_{2} x
$$

for some values of $a_{2}$ in $\mathrm{mG} / \mathrm{cm}$.

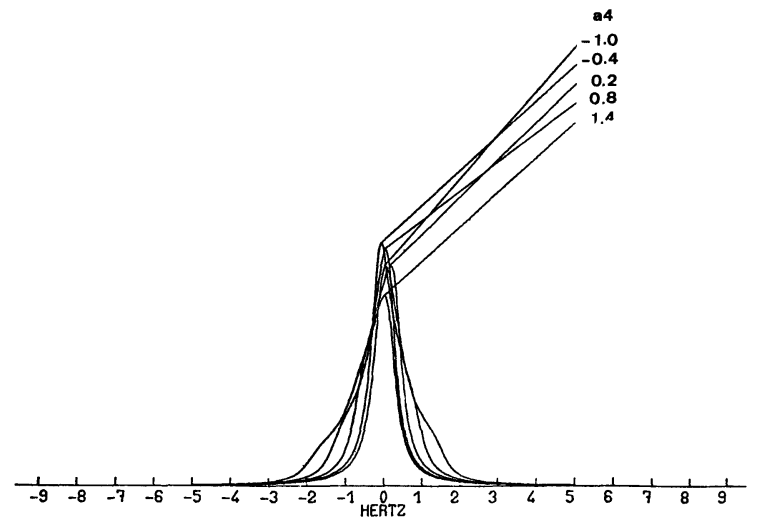

Fig. 8. - The magnetic field has in this case the form :

$$
H_{z}=H_{0}+0.25 z+a_{4}\left[z^{2}-\frac{1}{2}\left(x^{2}+y^{2}\right)\right],
$$

with $a_{4}$ in $\mathrm{mG} / \mathrm{cm}^{2}$.

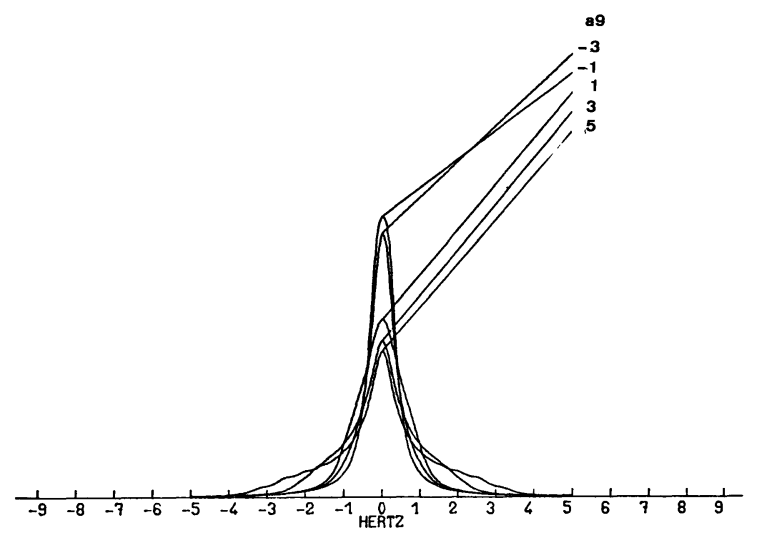

Fig. 9. - The magnetic field has the form :

$$
H_{z}=H_{0}+0.25 z+a_{9}\left[z^{3}-\frac{3}{2} z\left(x^{2}+y^{2}\right)\right]
$$

with $a_{9}$ in $\mathrm{mG} / \mathrm{cm}^{3}$.

5. Resolution enhancement. - The inhomogeneity of the magnetic field is the most important defect, in high resolution N.M.R. spectroscopy, in compounds, where the natural width of the spectrum has always been hidden by the artificial width produced by the detection device.

The influence of the magnetic field can sometimes be suppressed by an appropriate technique : for instance a double Fourier transform along a selected axis can be used [10], or the technique of the $J$ spectrum for homonuclear coupling [11]; this latter technique can be regarded as a particular application of the first one. However, it is the unidimensional Fourier transform technique which is used at present in most cases, especially for broad spectra, because the interpretation is easier. The best proof of this is found in the many empirical methods $[12,13,14]$ involving the particular shape of the free induction decay signal, used to counteract the influence of the inhomogeneous magnetic field. 
The systematic study, of the free induction decay signal affected by an inhomogeneous magnetic field can lead to a new method for the resolution of magnetic spectra. This non-empirical method is linked to the theoretical aspect of the physical spectrum.

All the other techniques which theoretically give a lorentzian absorption curve can be reduced to this case by an inverse Fourier transform. We mention for instance the slow field or frequency sweep technique, or the stochastic spectrometer technique [15].

We can use equation (3) or equation (5) for a nonspinning sample or equation (19) or equation (20) for a spinning sample.

All these equations are similar and they will be used in the same way to try and improve the resolution, i.e. :

$$
\bar{M}(t)=j k \mathrm{e}^{-t / T_{2}} \int_{V} g(v) \mathrm{e}^{-j[\Delta \omega+\gamma h(v)] t} \mathrm{~d} v
$$

in which $h(v)$ replaces $h_{z}(v)$ or $h_{0}(v)$ and where $g(v) \geqslant 0$ is practically equal to one in the irradiated volume $V$ of the sample and zero elsewhere.
5.1 THE INHOMOGENEITY FUNCTIONS. - In the case of a spectrum composed of many lines the signal can be written :

$$
\bar{M}(t)=j\left[\sum_{n=0}^{N} K_{n} \mathrm{e}^{-t / T_{2} n} \mathrm{e}^{-j \Delta \omega_{n} t}\right] \int_{V} g(v) \mathrm{e}^{-j \gamma h(v) t} \mathrm{~d} v
$$

the term $\int_{V} g(v) \mathrm{e}^{-j \gamma h(v) t} \mathrm{~d} v$, does not depend on the index $n$ and can be extracted from the formula. It depends only on the inhomogeneity of the field and on the shape of the sample and can be called the inhomogeneity function. It deteriorates all the lines of the spectrum in the same way.

If, in a given spectrum, we can isolate this inhomogeneity function we can then calculate the theoretical spectrum in an homogeneous magnetic field.

By separating the real and the imaginary parts of the inhomogeneity function we can write the two similar expressions :

$$
\int_{V} g(v) \cos (\gamma h(v) t) \cdot \mathrm{d} v-j \int_{V} g(v) \sin (\gamma h(v) t) \cdot \mathrm{d} v=A(t)-j B(t)
$$

or

$$
R(t) \mathrm{e}^{-j \varphi(t)} \text { with } R(t)=\sqrt{A^{2}+B^{2}} \text { and } \varphi(t)=\operatorname{arctg} \frac{B}{A} .
$$

5.2 THE INITIAL SIGNAL. - The spectrometer gives two signals out of phase by $\pi / 2$ :

$$
\begin{aligned}
M_{y}(t) & =\sum_{n=1}^{N} K_{n} \mathrm{e}^{-t / T_{2 n}}\left[A(t) \cos \Delta \omega_{n} t-B(t) \sin \Delta \omega_{n} t\right] \\
& =\sum_{n=1}^{N} K_{n} \mathrm{e}^{-t / T_{2 n}} R(t) \cos \left[\Delta \omega_{n} t+\varphi(t)\right]
\end{aligned}
$$

and

$$
M_{x}(t)=\sum_{n=1}^{N} K_{n} \mathrm{e}^{-t / T_{2 n}} R(t) \sin \left[\Delta \omega_{n} t+\varphi(t)\right] .
$$

The complex Fourier transform of $M_{x}(t)+j M_{y}(t)$ gives the complex spectrum :

$$
M(\omega)=\sum_{n=1}^{N} K_{n} \int_{V} \frac{T_{2 n}}{1+j T_{2 n}\left[\Delta \omega_{n}-\omega+\gamma h(v)\right]} \mathrm{d} v=R(\omega)+j I(\omega) .
$$

If the free induction decay signal was defined for all the time values $t \geqslant 0$ the real part $R(\omega)$ and the imaginary part $I(\omega)$ of the spectrum would be Hilbert transforms each other satisfying the causality principle :

$$
R(\omega)=\frac{1}{\pi \omega} * I(\omega) \text { and } I(\omega)=-\frac{1}{\pi \omega} * R(\omega)
$$

or, as the signal is sampled with points $n$ :

$$
R\left(\omega_{n}\right)=S_{n} * I\left(\omega_{n}\right)=\sum_{m=-\infty}^{+\infty} I\left(\omega_{m}\right) \cdot S_{n-m}
$$

and a similar expression for $I\left(\omega_{n}\right) . S_{m}$ is given by

$$
S_{m}=\frac{2}{\pi m} \sin ^{2} \frac{\pi m}{2}
$$

$m$ is an integer defined as $n:$ this is the discrete Hilbert transform [16].

In fact the time is limited to values from 0 to $T$ and the signal can be considered as being periodical with period $T$. The Fourier transform of this signal will also be periodical with period $(N-1) / T$ and sampled at intervals of $1 / T$. As we can no longer 
consider the free induction decay signal as zero for $t<0$, the relations linking the real and imaginary parts of the Fourier transform no longer exist and these two signals become independent and periodical. The discrete Fourier transform gives only $N / 2$ independent values for the real and the imaginary parts of the initial signal defined by $N$ points.

We can however deal with this situation by extrapolating the discrete function defined by $N$ points by means of $N$ additional zeros at least. Bartholdi and Ernst [17] have then shown that the real and imaginary parts of the discrete Fourier transform are again linked by a new relation, similar to the one needed for the causality principle. This known as the discrete and periodical Hilbert transform :

$$
\begin{aligned}
& R_{n}=I_{n} * S_{n}=\sum_{m} I_{m} \cdot S_{n-m} \\
& I_{n}=-R_{n} * S_{n}=\sum_{m} R_{m} \cdot S_{n-m}
\end{aligned}
$$

$n$ and $m$ are defined with at least $2 N$ points. Bartholdi and Ernst (it) have shown that $S_{m}$ is given by :

$$
S_{m}=\frac{1}{N} \sin ^{2} \frac{\pi m}{2} \cdot \operatorname{cotg} \frac{\pi m}{2 N} .
$$

5.3 Calculation OF THE INHOMOGENEITY FUNCTIONS. - If it is possible to isolate a single line in the spectrum, so that it can be considered as being independent of the other lines, then we can write, omitting the index $n$ to simplify the expression :

$$
M_{R}(\omega)=K \int_{V} \frac{T_{2}}{1+j T_{2}[\Delta \omega-\omega+\gamma h(v)]} \mathrm{d} v .
$$

By inverse Fourier transform we obtain

$$
M_{y R}(t)-j M_{x R}(t)
$$

with :

$$
\begin{aligned}
& M_{y R}(t)=K \mathrm{e}^{-t / T_{2}}[A(t) \cos \Delta \omega t-B(t) \sin \Delta \omega t] \\
& M_{x R}(t)=K \mathrm{e}^{-t / T_{2}}[A(t) \sin \Delta \omega t+B(t) \cos \Delta \omega t] .
\end{aligned}
$$

By introducing the vectors :

$$
\mathbf{E}=\left|\begin{array}{c}
A \\
B
\end{array}\right| \text { and } \quad \mathbf{M}_{R}=\left|\begin{array}{c}
M_{y R} \\
M_{x R}
\end{array}\right|
$$

we can also write

$$
\mathbf{M}_{R}(t)=K \mathrm{e}^{-t / T_{2}} \mathcal{R}_{\Delta}[\mathrm{E}(t)]
$$

in which $\mathcal{R}_{\Delta}$ is the rotation operator with an angle $\Delta \omega t$.

The use of the inverse rotation operator $\mathcal{R}_{-\Delta}$ brings the isolated line to the origin of the frequencies. If we know $\Delta \omega$, we can write $\mathrm{e}^{-t / T_{2}} A(t)$ and $\mathrm{e}^{-t / T_{2}} B(t)$, then if we know $T_{2}$, we can deduce $A(t)$ and $B(t)$, and then $R(t)$ and $\varphi(t)$.
It is sufficient now to write :

$$
\bar{M}_{A}(t)=\frac{\bar{M}(t)}{R(t)} \mathrm{e}^{j \varphi(t)}
$$

to obtain the theoretical free decay signal

$$
\bar{M}_{A}(t)=j \sum_{n=1}^{N} K_{n} \mathrm{e}^{-t / T_{2 n}} \mathrm{e}^{-j \Delta \omega_{n} t}
$$

We can remark that, when $R(t)=0$ the moment $\bar{M}(t)$ is also zero and we have $\bar{M}_{A}(t)=\frac{0}{0}$. But the number of points where $R(t)$ is zero is very small and we can easily calculate a value for $\vec{M}_{A}(t)$ by the extrapolation of two neighbouring points so introducing a negligible error. In fact, in many practical cases the function $R(t)$ does not have any zeros when $0 \leqslant t \leqslant T$.

We can notice too, that if the time $T$ is not long enough, then the function $\bar{M}_{A}(t)$ has non negligible values for $t$ close to $T$ and the Fourier transform produces some wiggles in the spectra.

\subsection{Calculation of $\Delta \omega .-$ We have :}

$$
\begin{aligned}
M_{R}(\omega) & =K \int_{V} \frac{T_{2}}{1+T_{2}^{2}[\Delta \omega-\omega+\gamma h(v)]^{2}} \mathrm{~d} v+ \\
& +j K \int_{V} \frac{T_{2}^{2}[\Delta \omega-\omega+\gamma h(v)]}{1+T_{2}^{2}[\Delta \omega-\omega+\gamma h(v)]^{2}} \mathrm{~d} v
\end{aligned}
$$

If there are no defects $(h=0)$ or if the spatial function $h(v)$ is odd along one of the coordinates, and if the origin of these coordinates is located at the symmetry center of the sample, then the imaginary part of $M_{R}(\omega), M_{x R}(\omega)=0$ when $\omega=\Delta \omega$.

For an even function of the field-defect and for a sample not centered exactly at the origin of the coordinates, there is a drift of all the lines. But we can see that this drift is relatively small. We then can calculate a value $\Delta \omega_{0}$ by solving the relation

$$
M_{x R}(\omega)=0 .
$$

The error is not important as in fact we introduce an unknown drift term $\Delta \omega_{1}=\Delta \omega_{0}-\omega$, which is small and constant. We have the same result if we consider that the defect of the magnetic field $h(v)$ has a constant term $h_{0}$ in its expansion. The shape of the lines is not changed and the relative distance between them is not modified.

5.5 Estimation OF THE $T_{2}$ PARAMETER. - In many experimental cases, this parameter can be introduced directly into the formulas because it is known beforehand. Indeed the only interesting relaxation time is that of the isolated line which can be used as a reference line placed in the experiment for this purpose.

A defect error affecting this parameter gives $A(t)$ and $B(t)$ an additional exponential and all the lines 
of the spectrum remain lorentzian, but with a larger width than that of the natural lorentzian shape.

An excess error produces over corrected lines. In this case there is also a prohibitive increase in the random noise, because we truncate in time the free induction decay signal.

When the reference line is chosen among the lines of the sample itself we can obtain $T_{2}$ by the usual methods such as, for instance from the slope at the origin.

But we must also take into account the value $1 / T$ of the Fourier transform step which limits the possible value of $T_{2}$. We cannot have a linewidth inferior to $1 / T$ if the line is defined by two points only. The interpolation does no improve the resolution. Consequently the limiting value $2 T$ is sometimes used for $T_{2}$ because larger values cannot bring any resolution improvement.

5.6 ISOLATION OF THE REFERENCE LINE. - After the isolated line which will be used as the reference line has been selected, it must be extracted from the totality of the spectrum with as little alteration as possible. An ideal filter cannot be used even if it is improved by a decrease of the absorption mode curve as $1 / x^{2}$ and of the dispersion mode curve as $1 / x$, because the period of the Fourier transform is not taken into account. This periodicity must be recreated.

A computation program can allow for this without altering the central part of the line shape and only the sides of the curve as adjusted.

5.7 THE INVERSE Fourier TRANSFORM. - The discrete inverse Fourier transform of this isolated signal should give the free induction decay signal of this isolated line. In fact this is not what happens because the above computation program, which has no great effect on the signal, does not indicate if the real and imaginary parts are discrete and periodic Hilbert transforms of each other. We will have a non zero signal for $t<0$, or, because of the periodicity, for the points near $2 N-1:$ the inverse signal will not have the form of an experimental free induction decay signal.

To correct this, the inverse Fourier transform has to be modified after the isolation of the reference line. If $R(n)$ is the absorption mode of this line we can obtain a dispersion mode by a Hilbert transform noted $T H: I^{\prime}(n)=T H(R(n))$. In the same way for $I(n)$ we can obtain $R^{\prime}(n)$ by $R^{\prime}(n)=T H(I(n))$. We have now four signals with :

$$
R(n)+R^{\prime}(n)=T H\left(I(n)+I^{\prime}(n)\right) .
$$

We can then by this operation recreate a free induction decay signal which satisfies the causality principle for periodic discrete functions. It must be noted that $R^{\prime}(0)$ is defined by $\sum_{n=0}^{2 N-1} R(n)$.
With this technique the free induction decay signal will have zero values for $n>N-1$.

We have shown [1] that this calculation, because of the parity of the functions and of the interpolation by at least $N$ zeros, is the same as the following one.

The inverse Fourier signal $M^{i}(k)$ defined for $0 \leqslant k \leqslant 2 N-1$ is replaced by the signal

$$
M(n)=M_{y}(n)-j M_{x}(n) \quad \text { with } \quad 0 \leqslant n \leqslant N-1
$$

defined by :

$$
\begin{aligned}
& M_{y}(n)=M_{y}^{i}(n)+M_{y}^{i}(2 N-n) \\
& M_{x}(n)=M_{x}^{i}(n)-M_{x}^{i}(2 N-n) .
\end{aligned}
$$

This method shortens the computation time and with it we have obtained, by using the totality of the spectrum, a reference line which is affected by the inhomogeneity of the magnetic field and which satisfies the relation for discrete functions.

5.8 RANDOM NOISE. - The random noise of the initial signal will also be processed by means of the inhomogeneity functions.

Remenbering that these functions are decreasing, we will conclude that the random noise for large $t$ values greatly increases.

Furthermore, when the initial free induction decay signal sink into the noise for large $t$ values, the application of $R(t)$ gives a noisy signal which can be very important. We can however considerably remedy this defect.

The envelope of the signal cannot be larger than an exponential $\mathrm{e}^{-t / T_{0}}$ in which $T_{0}$ can be the maximal relaxation time $T_{2}$ of the spectrum. Practically, we can choose as $T_{0}$ the $T_{2}$ used to define the inhomogeneity function of the maximum value of $T_{2}$ defined by the sampling rate of the functions.

The increase in the noise is then very much minimized, without greatly perturbating the resolution enhancement because the initial signal was not defined for these large $t$ values. A general effect remains which is similar to the truncation of the curve as noted above in $\S 5.3-5.4$.

It is finally this effect which creates the main noise/signal enhancement during the calculation of the resolution enhancement if we can also neglect the round-off errors of the computation. Then the increase of the noise is directly proportional to the increase of the real or chosen relaxation time $T_{2}$ of the reference line.

In this method of resolution enhancement, we have entirely separated the influence of the inhomogeneity of the magnetic field, which does not give a lorentzian curve, from the influence of the relaxation time which gives a lorentzian curve. So we can limit the lorentzian resolution, and consequently the noise, by choosing a shorter $T_{2}$, without perturbing the improvement of the line shape by means of the inhomogeneity functions : the lines of the spectrum 
remain lorentzian and, in particular, the base of the lines, deformed essentially by the magnetic field, remains on a straight line.

This arbitrary choice of $T_{2}$ is not a real inconvenience because we already know that the value of the relaxation time is limited by the number of sampled points and, in almost every case, will not be the real time $T_{2}$ of the reference line. We can then adjust $T_{2}$ in relation with the initial sensitivity of the spectrometer.

5.9 Some EXAMPLes. - The figures $10 a$ to $14 c$ show some results obtained by this method of resolution enhancement.

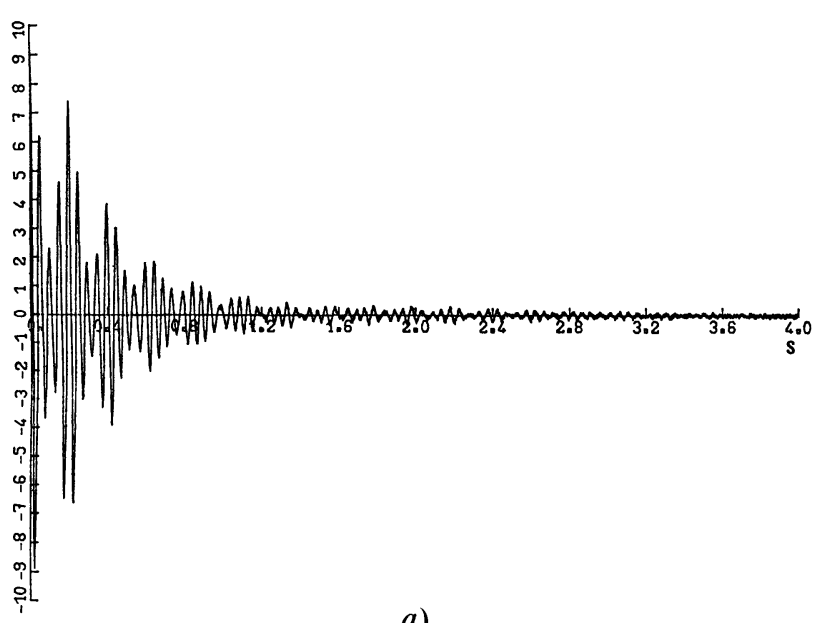

a)

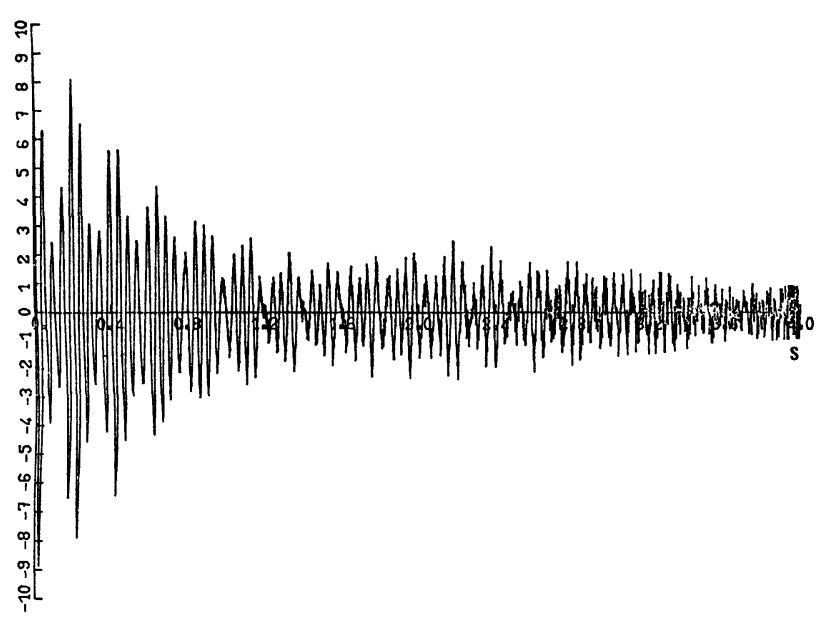

b)

Fig. 10. - Free induction decay signal with a good sensibility. a) Before resolution enhancement. b) After resolution enhancement. The noise is increased only for $t$ close to $T=4 \mathrm{~s}$.

5.10 SOME POSSIBLE USES OF THE INHOMOGENEITY FUNCTIONS. - We suggest here some possible applications, which have not been worked out yet, of the inhomogeneity functions.
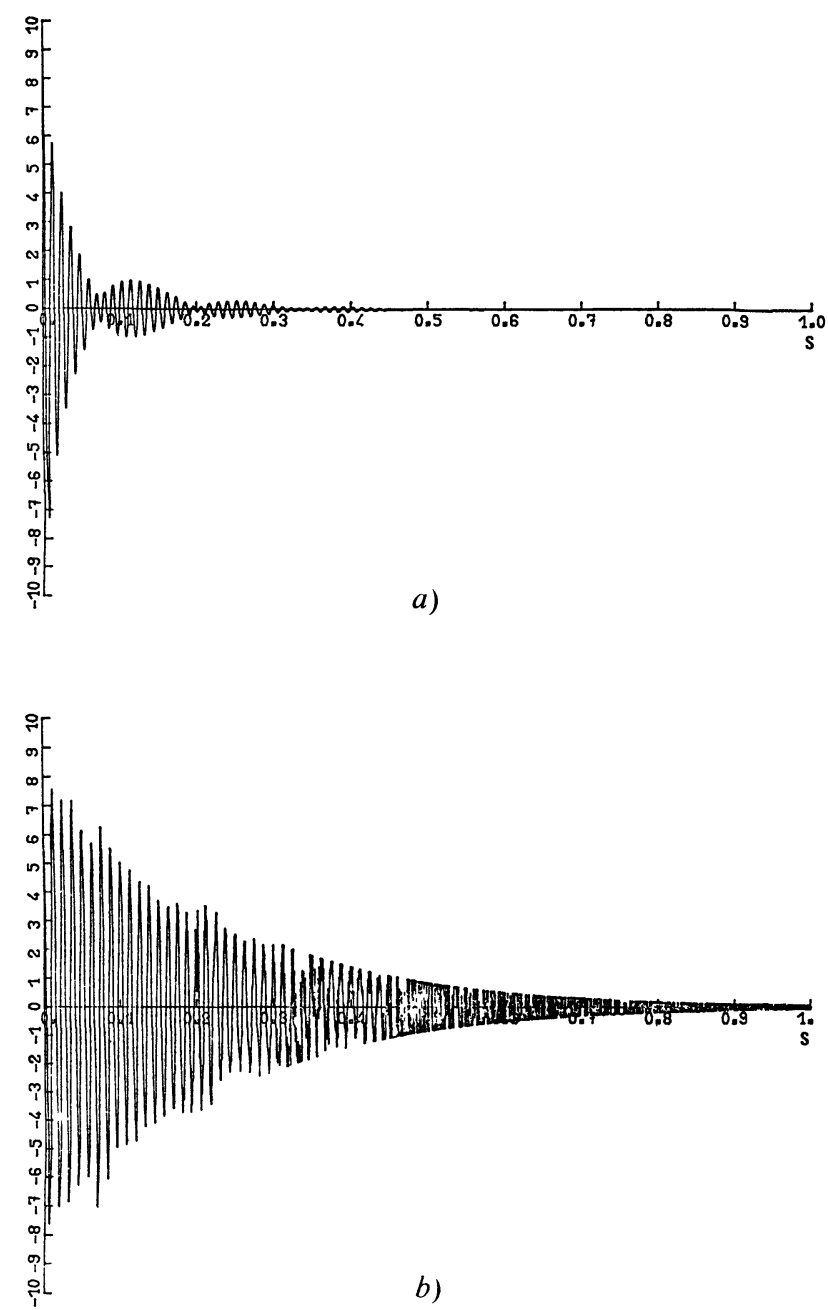

Fig. 11. - Free induction decay signal with a bad sensitivity. The inhomogeneity of the magnetic field has been very much increased on purpose : line one of the $\mathrm{CHCl}_{3}$ is split into two components. a) Initial free industion decay. b) Free induction decay after resolution enhancement with $T_{2}=1.3 \mathrm{~s}$. In this special case we have limited the increase of the noise for large $t$ values by the exponential $\mathrm{e}^{-2 \pi t / 1.3}$. In these two cases, the information is limited for $t \lesssim 0.45 \mathrm{~s}$, but in $b$ the signal is truncate for $t \approx 0.45 \mathrm{~s}$.

We have seen that we first introduce two functions $\mathrm{e}^{-t / T_{2}} A(t)$ and $\mathrm{e}^{-t / T_{2}} B(t)$ in which

$$
A(t)=\int_{V} g(v) \cos \gamma h(v) t . \mathrm{d} v
$$

and

$$
B(t)=\int_{V} g(v) \sin \gamma h(v) t \cdot \mathrm{d} v
$$

are the inhomogeneity functions. As $A(t)$ and $B(t)$ are related by a mathematical Hilbert transform, we can think of a new iterative method to measure the $T_{2}$ time of the reference line.

We can notice too that the limit for the $A(t)$ value for an homogeneous magnetic field is the volume $V$ of the irradiated sample, while it is zero for $B(t)$. 


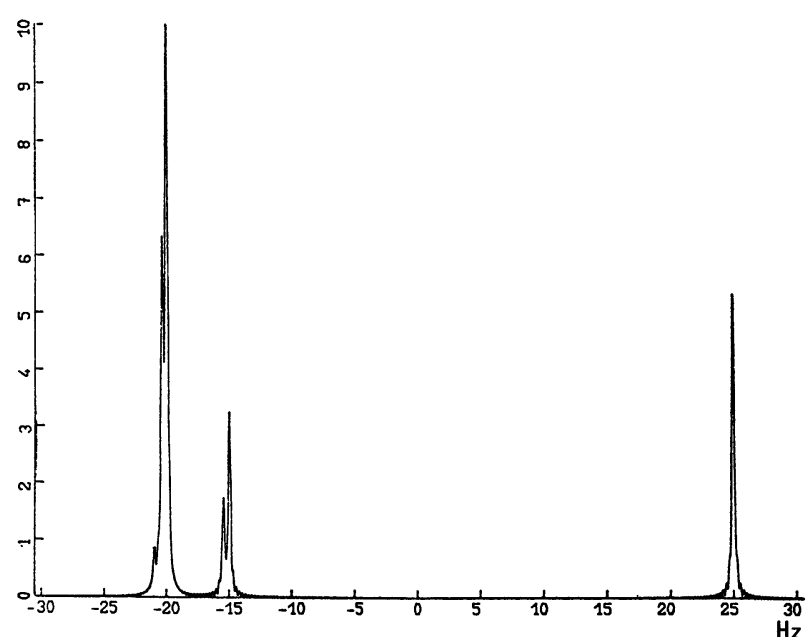

a)

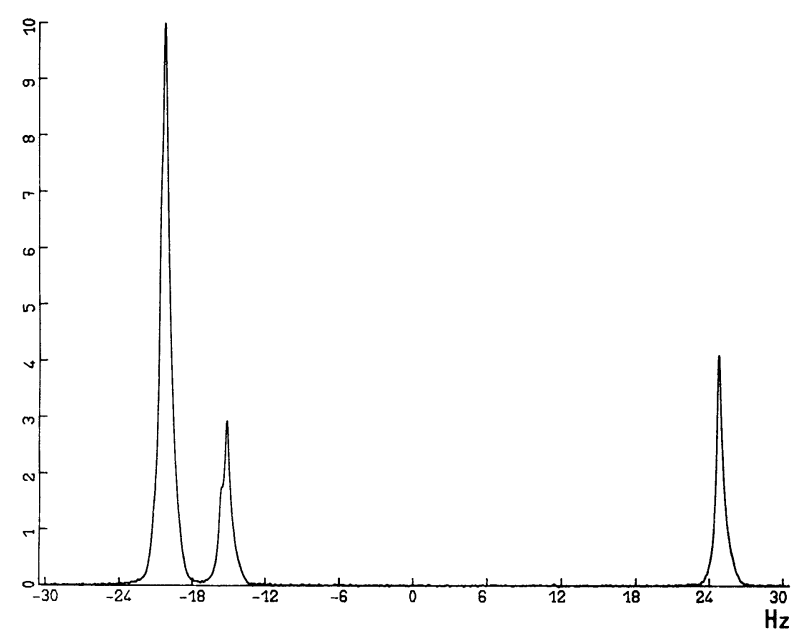

b)

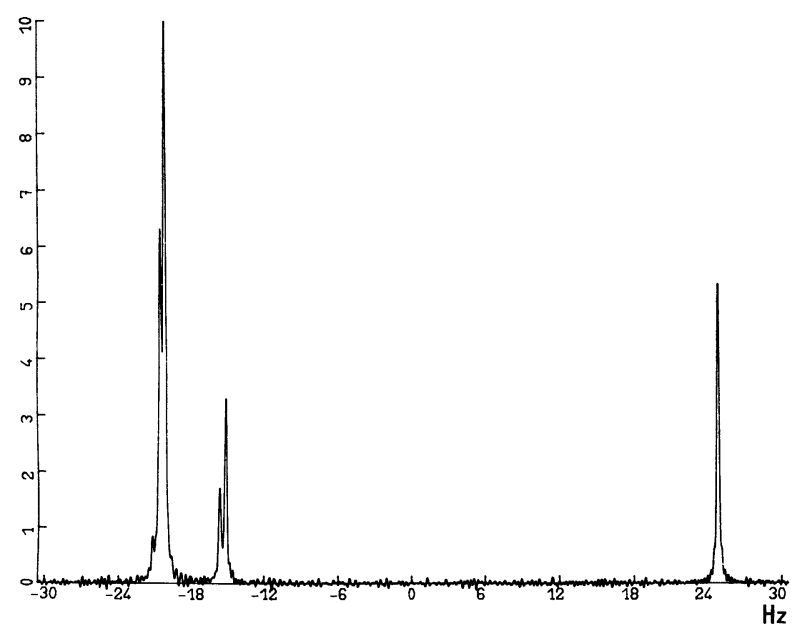

c)

Fig. 12. - Theoretical example for which the inhomogeneities have been calculated. a) Theoretical spectrum without defects in the magnetic field. $b$ ) Theoretical absorption spectrum for a sample with seven lines in an inhomogeneous field. The single line on the right will be used as the reference line. $c$ ) Spectrum of the figure $b$ after resolution enhancement.
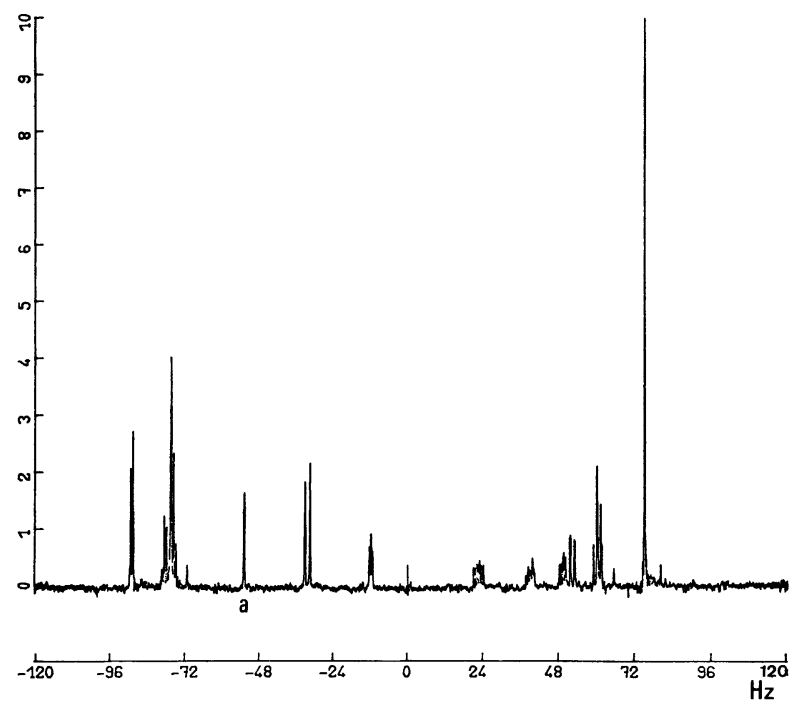

a)

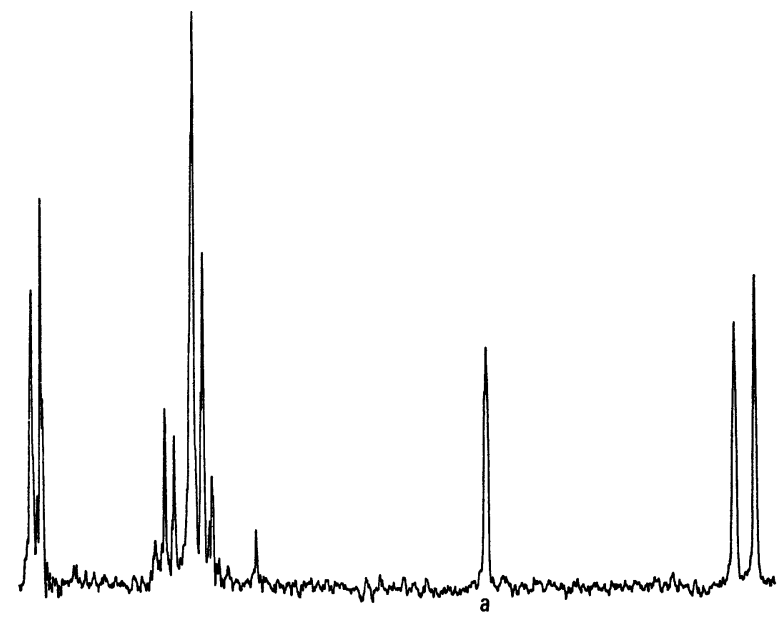

b)

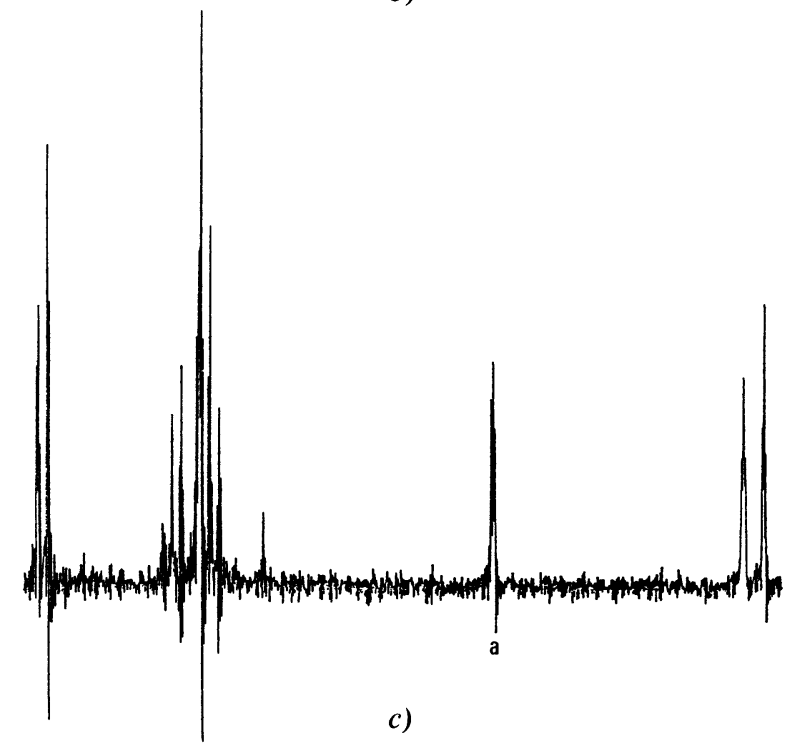

Fig. 13. - Improvement on a triplet of the collybolide. The reference line is that due to the $\mathrm{CH}_{3}$ located on the right. a) Collybolide spectrum. Only the part located in $a$ is studied. $b$ ) Left part of the spectrum : one line can be seen. $c$ ) After digital resolution enhancement, a triplet can be seen. The resolution cannot be better than 1.2 hertz in this experiment because of the number of steps in the data acquisition ( 2048 points on $2400 \mathrm{~Hz}$ ). 


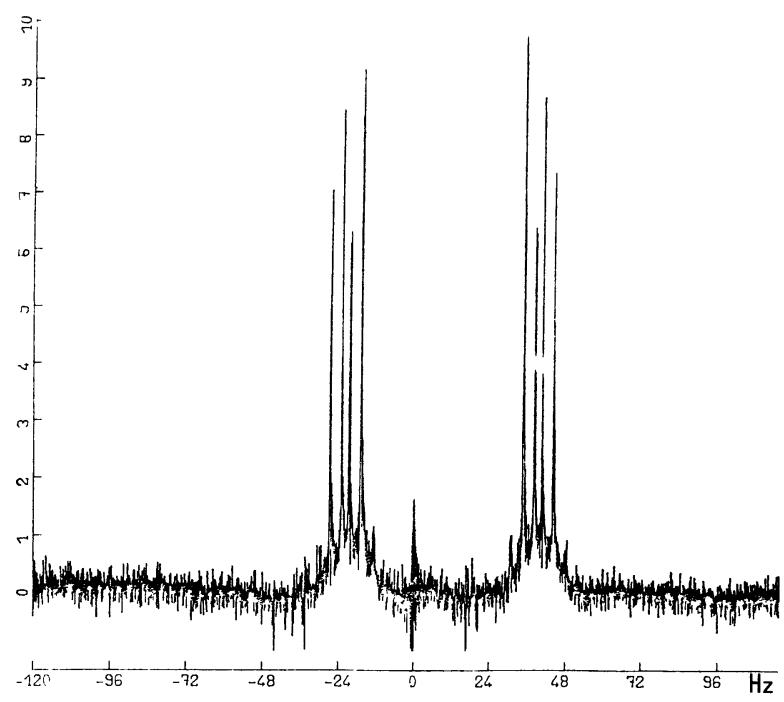

a)

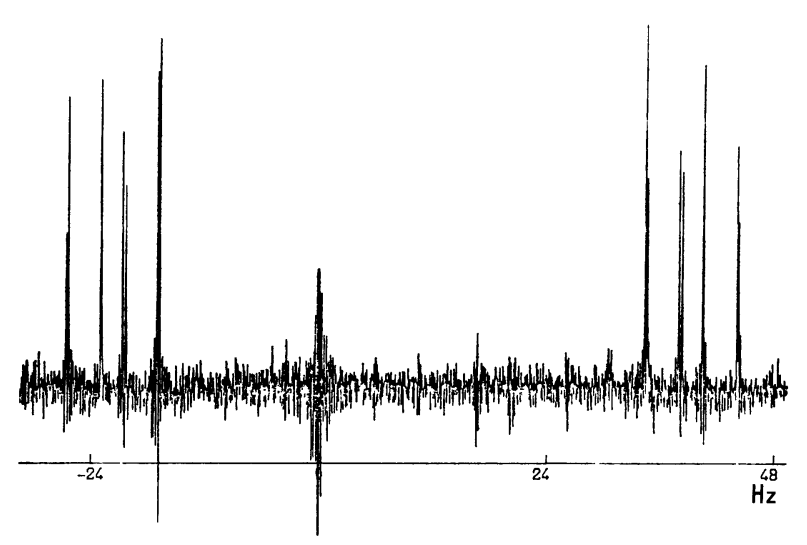

b)

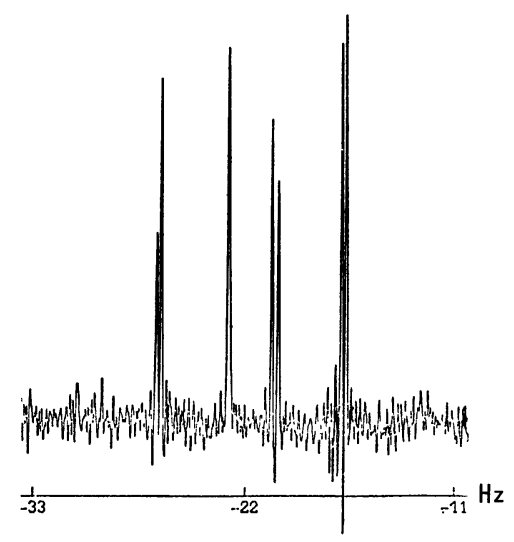

c)

Fig. 14. - a) Ortho-dichloro-benzen (O.D.C.B.). The reference line (T.M.S.) is located much further on the right. The wide base of the lines can be noticed. b) Same spectra broadened after resolution enhancement. The base of the lines is right. c) Left part of the preceding spectrum. The distance between the extreme doublets is about 0.18 hertz.
If there is a slight error on $\Delta \omega$ we obtain the complex function,

$$
\int_{V} g(v) \mathrm{e}^{j\left[\Delta \omega_{1}+\gamma h(v)\right] t} \mathrm{~d} v
$$

After treatment by these functions, we obtain a new spectrum in which all the lines are lorentzian, but with a shift $\Delta \omega_{1}$. A similar treatment of the new spectrum, with the same value of $\Delta \omega_{0}$ chosen for $\Delta \omega$, will give inhomogeneity functions in which $\gamma h(v)$ will have disappeared but which can show the preceding shift $\Delta \omega_{1}=\Delta \omega_{0}-\Delta \omega$.

It is also obvious that these functions which only depend on the shape of the sample, allow the magnetic field to be studied at a point.

For a spinning sample the side bands affected by these functions might also make a study of the field in the resonant cavity [1] possible.

6. Conclusion. - The Bloch equations with an inhomogeneous magnetic field developed near a spatial point in the sample enable us to draw the observed line shape of a high resolution N.M.R. experiment when the theoretically line slope in an homogeneous field would be a lorentzian curve. The study was made on a non spinning sample for many experiments, and on a spinning sample for the study of the free induction decay.

From these results, we can define a complex function

$$
\int_{V} g(v) \mathrm{e}^{j \gamma h(v) t} \mathrm{~d} v
$$

depending only on the shape of the sample and on the inhomogeneity of the magnetic field; this function can easily be derived from the general expression of the signal and can, no doubt, be very usefully applied to the treatment of a N.M.R. signal.

Although it is particularly well adapted to the processing of the signals of Fourier transform spectrometers, the great versatility of this function, which does not depend on the detection method, makes it highly suitable each time the influence of the inhomogeneity of the magnetic field has to be attenuated.

The most interesting application seems to be the resolution enhancement of a spectrum.

The main interest of this method to improve the resolution of a FTNMR signal by the use of a computer technique is that it is not an empirical method. The inhomogeneity of the magnetic field is treated as a second signal detected at the same time as the first, these two signals being separated afterwards. So we obtain, together with the theoretically perfect, spectrum, two signals which only depend on the inhomogeneity of the magnetic field and on the shape of the sample. These signals can be used to analyse the various components of the field in the sample. 
The measurement of one parameter only, namely the relaxation time $T_{2}$, remains necessary, although it is not very important for the calculation. It is often known from the conditions of the experiment, for instance if the reference line is that of a compound introduced for this purpose in the studied sample.

This method is affected only by two critical experimental parameters. First, the initial sensibility of the spectrometer (the given signal must not be strongly affected by noise) and second, the number of points in the sampling of the discrete functions which limits the limiting resolution.

Acknowledgment. - I wish to thank MM. Grivet and Sauzade in whose laboratory this work was carried out and also M. Bertein for the very fruitful discussions which greatly helped me in this work.

\section{References}

[1] TaQuin, J., Thesis, Orsay (1977).

[2] ERnst, R. R. and Anderson, W. A., Rev. Sci. Instrum. 37 (1966) 1.

[3] Jones, D. E., Thesis, Berkeley (1970).

[4] Jones, D. E. and Sternlicht, H., J. Magn. Reson. 6 (1972) 167.

[5] Taquin, J., C. R. Hebd. Séan. Acad. Sci. 283B (1976) 257.

[6] TAQUIN, J., Meth. Phys. d'Analyse (GAMS) 1 (1971) 3.

[7] ERNSt, R. R., Rev. Sci. Instrum. 30 (1974) 7.

[8] Harworth, O. and Richards, R. E., in Progress in N.M.R. spectroscopy (Pergamon Press, Eds) 1 (1966).

[9] Taquin, J., C. R. Hebd. Séan. Acad. Sci. 284B (1977) 145.

[10] Aue, J. P., Bartholdi, E. and Ernst, R. R., J. Chem. Phys. 64 (1976) 2229
[11] Freeman, R. and Hill, H. D. W., J. Chem. Phys. 54 (1971) 301.

[12] Campbell, I. D., Dobson, C. D., Williams, R. J. P. and LusebrinK, T. R., J. Magn. Reson. 11 (1973) 172.

[13] Monz, W. B., Poranski, C. E. and Sojka, S. A., J. Magn. Reson. 12 (1973) 214 and 13 (1974) 110.

[14] De Marco, A. and Wüthrich, K., J. Magn. Reson. 24 (1976) 201.

[15] ERnst, R. R., J. Magn. Reson. 3 (1970) 10.

[16] ERnst, R. R., J. Magn. Reson. 1 (1969) 7.

[17] Bartholdi, E. and ERnSt, R. R., J. Magn. Reson. 11 (1973) 9. 